\title{
OPERATOR REPRESENTATIONS OF $K$-FRAMES: BOUNDEDNESS AND STABILITY
}

\author{
Miao He, Jinsong Leng, Dongwei Li And Yuxiang Xu
}

\begin{abstract}
In this paper, a $K$-frame $\left\{f_{k}\right\}_{k \in Z}$ for a Hilbert space $H$, with the form $\left\{T^{k} f_{0}\right\}_{k \in Z}$ for an operator $T$ is analyzed. Some conditions under which a $K$-frame can be represented by an operator and then investigate the properties of this operator are discussed. More Specifically, a necessary and sufficient condition for a $K$-frame that has an operator representation can be obtained by a $K$-dual. Furthermore, we find the boundedness of the operator $T$ has an integral relationship with the operator $K$ when a $K$-frame can be represented by an operator $T$. In addition, the stability of operator representation is studied. We prove that the stability and boundedness are preserved under certain restrictions on the perturbation condition. A pretty small perturbation will heavily affect the property of being representable by an operator if $\mu>0$, and an example is used to illustrate it. Furthermore, some elements from a subspace of $H$ are used to perturb a $K$-frame, and then some useful stability results are obtained.
\end{abstract}

Mathematics subject classification (2010): Primary 42C15; Secondary 46C07.

Keywords and phrases: Frames, $K$-frames, boundedness, stability; $K$-dual.

\section{REFERENCES}

[1] J. Duffin Richard, Albert C. Schaeffer, A class of nonharmonic Fourier series [J], Trans. Amer. Math. Soc. 1952, 72: 341-366.

[2] Dongwei Li, Jingsong Leng, Tingzhu Huang, On sum and stability of g-frames in Hilbert spaces, Linear and Multilinear Algebra, 2018, 66 (8): 1578-1592.

[3] Xiangchun Xiao, Yucan Zhu, L. Găvruţa, Some Properties of $K$-frames in Hilbert Spaces, Results in mathematics, 2013, 63 (3-4): 1243-1255.

[4] Jinsong Leng, Deguang Han, Tingzhu Huang, Probability modelled optimal frames for erasures, Linear Algebra and Its Applications, 2013, 438 (11): 4222-4236.

[5] Radu Balan, Peter G. Casazza, Dan Edidin, Gitta Kutyniok, A new identity for Parseval frames, Proceedings of the American Mathematical Society, 2007, 135 (4): 1007-1015.

[6] Roderick B. Holmes, Vern I. Paulsen, Optimal frames for erasures, Linear Algebra and Its Applications, 2004, 377 (1): 31-51.

[7] Deguang Han, Frame representations and Parseval duals with applications to Gabor frames, Trans. Amer. Math. Soc., 2008, 360 (6): 3307-3326.

[8] Xiangchun Xiao, Mingling Ding, Yucan Zhu, Several studies of continuous K-frame in Hilbert spaces, Journal of Anhui University, 2013 (2): 7.

[9] Peter G. Cazassa, Ole Christensen, Perturbation of operators and applications to frame theory, Journal of Fourier Analysis \& Applications, 1997, 3 (5): 543-557.

[10] Amir Khosravi, Musazadeh Kamran, Fusion frames and g-frames, [J], Journal of Mathematical Analysis and Applications, 2008, 342 (2): 1068-1083.

[11] L. GĂVRUŢA, Frames for Operators, Appl. comp. Harm. Anal. 2012, 32: 139-144.

[12] Ole Christensen, Has AnNASAB MARZIEH, Frame properties of systems arising via iterated actions of operators, Applied and Computational Harmonic Analysis, 2019, 46 (3): 664-673.

[13] Ole Christensen, Has annasab Marzieh, Operator representations of frames: boundedness, duality, and stability, Integral Equations and Operator Theory, 2017, 88 (4): 483-499. 
[14] Li Liang, The Optimal K-Duals for 1-Erasure for Parseval K-Frames, Advances in Applied Mathematics, 2014, 03 (4): 192-200.

[15] Miao He, Jingsong Leng, Yu Jiali, Dongwei Li, Probability Modeled Optimal K-frame for Erasures, IEEE Access, 2018, 6: 54507-54515.

[16] Bodmann Bernhard G., Vern I. PAulsen, Frame paths and error bounds for sigma-delta quantization, Applied and Computational Harmonic Analysis, 2007, 22 (2): 176-197.

[17] Balan Radu, P. Casazza, C. Heil, Z. Landau, Deficits and excesses of frames, Adv. Comp. Math, 2002, 18 (2-4): 93-116.

[18] Ole Christensen, Hasannasab Marzieh, Ehsan Rashidi, Dynamical sampling and frame representations with bounded operators, Journal of Mathematical Analysis and Applications, 2018, 463 (2): 634-644.

[19] Ole Christensen, An introduction to frames and Riesz bases, Second expanded edition, Applied and Numerical Harmonic Analysis. Birkhäuser Boston Inc., Boston, MA, 2016.

[20] Jinsong Leng, Deguang Han, Tingzhu Huang, Optimal dual frames for communication coding with probabilistic erasures, IEEE Trans Signal Process, 2011, 59 (11): 5380-5389.

[21] A. F. DANA, R. GowAIKAR, R. PALANKI, Capacity of wireless erasure networks, IEEE Transactions on Information Theory, 2006, 52 (3): 789-804.

[22] Deguang Han, Classification of finite group-frames and super-frames, Canad. Math. Bull., 2007, 50 (1): 85-96.

[23] Jinsong Leng, Deguang Han, Optimal dual frames for erasures II, Linear Algebra and its Applications, 2011, 435 (6): 1464-1472.

[24] G. Upenderreddy, N. Gopalreddy, B. Krishnareddy, Frame Operator and Hilbert-Schmidt Operator in Tensor Product of Hilbert Spaces, Journal of Dynamical Systems \& Geometric Theories, 2009, 7 (1): 61-70. 